\title{
Reflexiones preliminares para pensar la formación de formadores en la universidad moderna: Nuevas miradas y nuevos diálogos desde la complejidad
}

\author{
Preliminary Reflections to Think Training of Trainers in the Modern University: New Look \\ and New Dialogs from the Perspective of Complexity
}

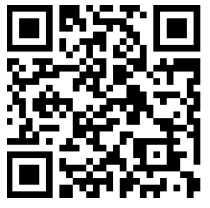

Recibido 22 de enero de 2016 • Corregido 15 de diciembre de 2016 • Aceptado 19 de abril de 2017
Jorge Ferrada-Sullivan ${ }^{1}$

Universidad de Los Lagos

Puerto Montt, Chile

jorge.ferrada@ulagos.cl

http://orcid.org/0000-0001-5966-6796

\begin{abstract}
Resumen: Con este artículo se pretende reflexionar sobre los principios epistemológicos que sustentan el pensamiento complejo Morin en su relación con los procesos formativos y pedagógicos al interior de las aulas chilenas. La trayectoria temática del texto pretende relacionar la vinculación directa entre las nociones fundamentales de la complejidad y la valoración de ciertos principios metodológicos activos que potencien la reflexividad y la experiencia del estudiantado. Los tres supuestos pedagógicos y basales de dicho vínculo se sustentan en las relaciones de diálogo con la diversidad contextual, en la recursividad de la experiencia y en el principio hologramático del conocimiento. El propósito del texto queda sustentado en la presentación de una serie de argumentos críticos que permitan tensionar las formas tradicionales de enseñanza y aprendizaje al interior de los espacios educativos. Conceptualmente la noción de complejidad permite discutir con la tradición educativa y con elementos de cambio para una reflexividad práctica en torno a los procesos formativos que portan y comportan nuevos paisajes cognitivos en torno a la enseñanza. La experiencia del aprendizaje, la autonomía creativa y el contexto actual serán fundamentos claves para futuras transformaciones sistémicas. Finalmente, se propone un conjunto de recomendaciones para la puesta activa de acciones metodológicas que podrían utilizarse en los diversos espacios educativos de Chile.
\end{abstract}

Palabras claves: Pensamiento complejo; principios pedagógicos; sujeto pedagógico; multidimensionalidad; acción-reflexión; pedagogía crítica contemporánea.

\footnotetext{
${ }^{1}$ Doctor en Filosofía, Magíster en Artes y Profesor de Historia y Geografía, Universidad de Chile. Profesor en áreas de las ciencias sociales como filosofía, epistemología, educación, estética y en seminarios de tesis e investigación en pregrado y postgrado. Además, en cargos directivos, supervisando procesos de gestión, desarrollo disciplinar y curricular. Recientemente jefe de la Carrera de Pedagogía en Artes, Universidad de Los Lagos, Puerto Montt y director de la Escuela de Educación de la Universidad Miguel de Cervantes. Participante de investigaciones en educación y en la realización de ponencias a nivel nacional e internacional, participante en congresos internacionales como: Escrituras para el Siglo XXI, Facultad de Humanidades, Universidad de Cuyo, Mendoza; Epistemología y Ciencias Sociales, realizado en la Universidad Nacional Autónoma de México (UNAM) y en el Epistemologías y Narrativas del Sur de la Universidad de Los Lagos. Posee experiencias en trabajos con comunidades culturales para el desarrollo local y en la creación de proyectos que permitan el surgimiento comunitario y social. Curador de exposiciones artísticas en la ciudad de Puerto Montt.
} 
doi: http://dx.doi.org/10.15359/ree.21-2.21

URL: http://www.una.ac.cr/educare

CORREO: educare@una.cr

\begin{abstract}
This article reflects on the epistemological principles underlying Morin's complex thought in their relation with the formative and pedagogical processes in Chilean classrooms. The paper theme aims to relate the direct connection between the fundamental notions of the concept of complexity and the evaluation of certain active methodological principles enhancing the reflexivity and the students' experience. The three basal and pedagogical assumptions of such connection are based on relations of dialog with the contextual diversity in the recursivity of the experience and in the hologramatic principle of knowledge. The purpose of the text is based on the presentation of critical arguments that allow tensioning the traditional ways of teaching and learning within the educational spaces. Conceptually, the notion of complexity allows the discussion with the educational tradition and with elements of change for a practical reflexivity on formative processes. These processes carry and imply new cognitive landscapes about teaching. The learning experience, the creative autonomy and the current context will be key foundations for future systemic transformations. Finally, a set of recommendations are proposed to implement active methodological actions that could be used in the various educational spaces in Chile.
\end{abstract}

Keywords: Complex thought; pedagogical principles; pedagogical subject; multidimensionality; action-reflection; contemporary critical pedagogy.

\title{
Introducción
}

Todo desarrollo verdaderamente humano significa desarrollo conjunto de las autonomías individuales, de las participaciones comunitarias y del sentido de pertenencia con los otros individuos. Es más, el sujeto, en tanto que sí mismo, pertenece a una comunidad y se desenvuelve de cierta manera en ella y a través de ella. Pertenece y le pertenece porque, de hecho, su identidad, en gran parte, ha sido conformada por el tipo de educación obtenida.

Nos-otros y los otros individuos en las culturas mantienen las identidades sociales y comunitarias y, por esto, la comunidad debiera retornar a su carácter cultural/histórico/ identitario, en la medida en que sus valores, costumbres, ritos, normas y creencias compartidas se han sostenido en la enseñanza y en los aprendizajes culturalmente socializados.

Un sistema pedagógico sustenta valores socio-políticos, culturales, éticos y estéticos instalados ya sea por métodos como por procedimientos que establecen (supuestamente) los ideales formativos trazados por el sistema educativo de cada nación. Por eso cada sociedad, en particular, necesita construir, en consistencia con los principios ideológicos y culturales, las bases sobre las que se sustenta el proceso de formación de la persona y la manera en que se deriva el logro del ideal de ser humano al que se aspira. En el caso de Chile, la educación escolar no ha presentado grandes cambios en los últimos años. Existen algunas barreras infranqueables que no han permitido avances sustanciales en el sentido y en la propiedad de los procesos formativos al interior de las aulas. Se siguen sustentando principios de equidad y calidad con parámetros de mercado; las reformas que ha impulsado el Estado no han reflejado mejoras en la calificación de los cuerpos docentes y los procesos de enseñanza y aprendizaje aún se sostienen en metodologías contenidistas y asignaturistas. Esto conlleva a pensar que el paradigma socioeducativo chileno no posee principios orientadores que determinen cambios 
sostenibles en el tiempo. Certidumbres concretas de aquello se evidencian en los bajos puntajes obtenidos por estudiantes de pedagogía en la prueba INICIA (Esta evaluación entrega un panorama sobre las condiciones y capacidades en que los sujetos egresados de pedagogía se aproximan al mundo del trabajo). Los resultados de la evaluación en los últimos cinco años indican, en términos generales, que un porcentaje mayoritario del futuro profesorado no maneja los contenidos que debe enseñar ni cuenta con las herramientas didácticas que se requieren.

Es en este sentido, ciertos elementos constituyentes del paradigma de la complejidad (Morin, 2011) permiten relacionar y construir una propuesta para la práctica efectiva en la formación pedagógica. Dicha práctica será posible por la puesta en valor y del reconocimiento de la diversidad de relaciones e inter-retro-acciones que se producen en el engranaje sujetomundo-lugar y que, por cierto, se reproducen en los espacios educativos. Todas las cosas son fenómenos que se comportan como sistemas entretejidos, susceptibles de ser transformados en nuevas matrices conceptuales correspondidas con nuevas miradas de la realidad cultural y educativa. Por esto mismo, retornar al pensamiento complejo y proyectar su práctica en las aulas tendería a inquietar las tradicionales perspectivas y puntos de vista que el Estado chileno posee para enfrentar las incertidumbres que se originan al interior del sistema educativo.

\section{Ontología de la complejidad: Evocar la experiencia aprendida}

En noviembre del año 1997, Edgar Morin fue invitado a participar, en un consejo científico, presidido por el entonces Ministro de Educación Nacional de Francia, para realizar ciertas sugerencias en el mejoramiento de la enseñanza secundaria. El interés de Morin no solo incluía dicha enseñanza, sino que también, su inquietud por todo el sistema educativo francés y, en especial, por la educación superior que formaba docentes de secundaria y del futuro. Desde esa reunión multidisciplinar, nació el texto antológico El desafío del siglo XXI. Unir los conocimientos (Morin, 1999a). El filósofo inaugura el contenido del libro con un ensayo introductorio que manifestaba la necesidad de volver a mirar los procesos educativos desde la perspectiva de la complejidad.

Puede apreciarse, sigilosamente, que se está iniciando en Occidente una transformación muy profunda a nivel cultural. Las ciencias sociales hablan de cambio de paradigma, la globalización, la era de la sociedad del conocimiento, internet y la multi-virtualidad, haciendo referencia a cambios muy profundos en las nuevas formas de relacionarnos y de pertenecer a nuestras comunidades educativas.

Estas breves reflexiones en torno a cuestiones vinculadas con el pensamiento complejo sitúan los nuevos desafíos, muchos de ellos pendientes para una educación en los diversos escenarios formativos de la realidad chilena. Una pedagogía sostenida en los lineamientos de este paradigma consiste, entonces, en la posibilidad que todo ser humano pueda seguir aprendiendo a lo largo de toda la vida, con aprendizajes de múltiples formas y de distintas fuentes que la realidad proporciona. Por esto mismo, cuando se logran desarrollar las capacidades 
doi: http://dx.doi.org/10.15359/ree.21-2.21

URL: http://www.una.ac.cr/educare

CORREO: educare@una.cr

de autoaprendizaje para desenvolverse en los contextos actuales, se torna fundamental el descubrimiento de la experiencia (conocimiento) y la autonomía de los aprendizajes como forma holística para la comprensión de la realidad.

Tradicionalmente, las ciencias han tendido a determinar su objeto y hacernos explicable el mundo. La formación que el profesorado realiza en las escuelas y universidades debe plantear, antes que nada, la pregunta, en tanto problemática y desafío: ¿qué ideal de ser humano queremos formar? ¿qué identidad lo sostiene? ¿a qué pertenece?, preguntas que se fundan en la experiencia, en el conocimiento, en la razón y en la instalación por parte de nosotros y nosotras, docentes de nuevos fundamentos pedagógicos.

No obstante, dentro de esta lógica queda poco espacio para la indeterminación, para el asombro y lo novedoso, para la incertidumbre. Desde esta perspectiva, debemos avanzar hacia la re-articulación de las disciplinas, pero lo más importante es mantener el espíritu crítico que permite descubrir sentidos nuevos de las cosas y sus fenómenos. Señala Pineau (2009) que cuando existe una apertura de los aprendizajes relacionados con una multidimensionalidad de los conocimientos, se plantean nuevos puntos de vista sobre las teorías educativas clásicas que reducían la formación educativa a una mera instrucción. Este reconocimiento de la necesidad de cambio en los aprendizajes diversos y permanentes tendría que situar el problema tradicional de la educación en nuestro país. Dicha necesidad radical se comprende en dos sentidos: el aprendizaje debiera ser presentado como el factor principal de la formación humana, suplantando factores ambientales, biológicos o sociales vistos anteriormente como condicionantes. Y, por otra parte, el aprendizaje se ve como un movimiento recursivo, reflexivo y permanente. Una suerte de evocación de la experiencia aprendida en los procesos de formación pedagógica (evocar, del latín evocāre) entendido como traer a la memoria algo vivido y percibido desde el pasado, recordaría una diversidad de escenas de remembranzas que dan el sustento a la compleja manera en que el sujeto es en el mundo).

La experiencia es devenir constante hacia el presente como una forma de hacer presencia "actualizada" del sentido vivido. El evocar se cruza con su alteridad presente en tanto presencia evidente de sus vínculos contextuales con la realidad. Su presencia vendría a ser correspondida con una presentación originaria, un verdadero acontecer del sentido mismo de la manera vivida como experiencia en el presente inmediato del mundo. Como sostiene Nancy (1993), la presencia no viene sin borrar la presencia que la representación querría designar (su fundamento, su origen, su sujeto). La venida es una venida y, a su vez, una partida que relaciona el sentido con el sujeto. Es un desde y un hacia, que en ningún sitio desborda el mundo hacia un principio o un final. "In that since and that toward within the limit of the world, is the whole world, is his coming, his coming to this in this" [En ese desde y ese hacia dentro del límite del mundo, está el mundo entero, es su venida, su venida a ello, en ello] (Nancy, 1993, 140). Se presenta y se nos presenta constantemente lo vivido como evocación en presencia y como una experiencia subjetiva del sentido de ser-en-el-mundo. La presencia -en este sentido experiencia- será 
siempre ella misma nacimiento y acontecimiento, la llegada que borra sus huellas y se retira a sí misma para su estancia en el sujeto presente (Derrida y Ferraris, 2009). Por esto mismo, regresar a los aprendizajes en la trayectoria formativa tendería a construir su episteme desde el acontecimiento de la experiencia del saber y del conocer que los actores del sistema educativo poseen. Una sala de clases será, sin duda, el espacio de experiencias que se constituyen en conocimientos (su venida a la distancia y a la presencia educativa y formativa de los saberes). Para Maturana (citado por Moraes y De La Torre, 2002):

Cuando priorizamos la formación del SER, teniendo como foco de atención principal su HACER, ya que el SER y el HACER también están imbricados. Potenciando el HACER estaremos, simultáneamente, potenciando el SER. Convidándolo a reflexionar sobre su acción, estaremos haciendo que desarrolle su autonomía, su creatividad y conciencia crítica. (p. 52)

El pensamiento complejo es una nueva impronta para las emergentes generaciones, para la universidad y para la educación chilena. Es complejo, porque debemos pensar como pensarlo y, por consiguiente, como enseñarlo. Todo conocimiento constituye, al mismo tiempo, una traducción y reconstrucción a partir de señales, signos, símbolos, en forma de representaciones, ideas, teorías, discursos. Esta base transforma el conocimiento en complejidad e incertidumbre. Como sostiene Morin (1983), conocer y pensar no es llegar a una verdad totalmente cierta, es dialogar con la incertidumbre. Obviamente, el contexto en el cual se desarrollan nuestros procesos de enseñanza aprendizaje y los que socializamos con nuestro estudiantado están, muchas veces, marcados por prácticas clásicas y descontextualizadas del momento histórico que vivimos. Por esto, debemos poner especial atención en las metodologías de enseñanza y de aprendizajes al interior de las escuelas formadoras. Consideremos, por un momento, que muchos de los actores del sistema educativo están pensando menos y haciendo más. Esto genera desconfianza en la práctica y en la calidad de los procesos involucrados. Pensar y hacer caminan juntos en los senderos de la innovación y cambio. Dejemos que su relación -placer y deseo a la vez- manifieste su dinamismo permanente y su existencia potencial al interior de las organizaciones educativas. Por este motivo, la vida humana, de la sociedad, de las instituciones, no pueden seguir siendo significadas como objetos cerrados y sin movimiento. Filosóficamente, la palabra existencia evoca movimiento y acción. La experiencia debiera sustentarse en la confianza, precisamente por el carácter ontológico, de su presencia en movimiento: el Ser está ahí en la medida en que el confiar es parte de su esencia. Los sujetos necesitan de los otros en tanto que forman un entre-tejido complexus (Morin, 1999b) en su relación especular y social, y en cuanto necesidad del otro: la evocación (traer a la presencia lo otro y el otro) se transforma en su responsabilidad. La educación actual debe ser la primordial puesta en certeza de nuevas prácticas que prioricen la seguridad de los procesos formativos. Se confía en que el profesorado destacará por su desempeño ya que este confía en las autoridades que conducen el camino de la educación. Cuando a todos los actores se les brinda confianza, entonces, querrán hacerse parte de ella. En otras palabras, hacernos dignos de la confianza que en cada protagonista 
doi: http://dx.doi.org/10.15359/ree.21-2.21

URL: http://www.una.ac.cr/educare

CORREO: educare@una.cr

se ha depositado: ¿será posible seguir sustentando el sistema educativo chileno en la vigilancia del cumplimiento? Por esto mismo, el carácter ontológico de este verdadero acontecimiento (lo experiencial) se transforma en la instancia donde la presencia del confiar $\mathrm{o}$, si se quiere, de aquella ontología de la complejidad construye los cimientos bases para una educación con sentido y en el sentido del proyecto formativo y educativo futuro. Para Maturana (2007, pp. 32-33):

¿Cómo puedo aceptarme y respetarme a mí mismo si estoy atrapado en mi hacer (saber) porque no he aprendido un hacer (pensar) que me permite aprender cualquier otro quehacer al cambiar mi mundo si cambia mi vivir cotidiano? Si la educación en Chile no lleva al niño chileno a un quehacer (saber) que tiene que ver con su vivir cotidiano de modo que pueda reflexionar sobre su quehacer y cambiar el mundo sin dejar de respetarse a sí mismo y al otro, la educación en Chile no sirve a Chile.

La razón práctica de la complejidad radica en que desde la formación inicial del profesorado se presentan los elementos configurantes de las competencias fundamentales para el ejercicio docente. Por esto mismo, ¿para qué seguir sometiéndoles a estrictas directrices, si gran cantidad ya es competente? Lo que es común a la mayoría recibe menos atención, pues los seres humanos se preocupan más de lo que individualmente les pertenece que de lo que poseen con otros en común. Junto con los otros individuos, y en la necesidad de considerar la legitimación de los otros, se nos aparece el ser de la complejidad, la humanidad en gloria y majestad: la entrega al otro ser me prepara para la alteridad. Acciones que sin duda se soportan en el amor al saber, en el amor a la enseñanza responsable y en una profesionalidad amorosa para la entrega pedagógica. Esta relación de alter-sentido formativo manifiesta la originaria manera en que el conocimiento sensibiliza las prácticas y los espacios educativos. Un sistema educativo basado en el desarrollo del pensamiento complejo para la acción no puede ser cuestionado ni menos estrictamente unívoco en sus prácticas.

La autenticidad del amor no está sólo en proyectar nuestra verdad sobre el otro, para finalmente no verlo más que través de nuestros ojos; está en dejarnos contaminar por la verdad del otro. No hay que ser como esos creyentes que encuentran lo que buscan porque proyectan la respuesta que esperan. Y ahí está también la tragedia: llevamos en nosotros tal necesidad de amor que, a veces, un encuentro en un buen momento -acaso en un mal momento- desencadena el proceso de la fulminación, la fascinación.

Enesemomento, proyectamos sobreotro esta necesidad deamor, la fijamos, la endurecemos e ignoramos al otro que se convierte en nuestra imagen, nuestro tótem. Loignoramos creyendo adorarlo. Ahí está, en efecto, una de las tragedias del amor: la incomprensión de sí y del otro. Pero la belleza del amor es la interpenetración de la verdad del otro en sí, de la de sí en el otro, es hallar la propia verdad a través de la alteridad (Morin, 1998, p. 6). 
Señala el francés Morin (1983) que la idea de sistemas abiertos, dinámicos, críticos y creativos introduce la idea de existencia, es decir, hay, a la vez, un dinamismo permanente, entre estabilidad, certeza y verdad e inestabilidad, fragilidad, desorden, riesgo y desaparición. El fracaso y la certeza, el temor y el riesgo se resisten a existir en un mundo con niveles competitivos exitistas. Sin embargo, estas relaciones dialécticas necesarias se transforman en una novedad cuando pensamos que el origen del cambio o de las transformaciones educativas manifiestan, sutilmente, las condiciones para la aparición de la novedad como acontecer.

La confianza y la seguridad que provocan la experiencia del conocimiento debieran ser la gran tarea docente para hacerse cargo de la existencia del otro como sujeto en formación. Lo vinculante de la relación del sí mismo o misma hacia el otro u otra no correspondería a un hecho fortuito -que sin duda podría maquillar las actuales salas de clases-, más bien el otro sujeto viene hacia el sí mismo, porque ha aceptado su presencia y la necesidad del otro ser como condición de su propia existencia. Una suerte de alteridad de la responsabilidad del acto educativo. Para Lévinas (1991), la responsabilidad:

No es un simple atributo de la subjetividad, como si esta existiese ya en ella misma, antes de la relación ética. La subjetividad no es un para sí; es, una vez más, inicialmente para otro. El otro no es próximo a mí simplemente en el espacio, o allegado como un pariente, sino que se aproxima esencialmente a mí en tanto yo me siento -en tanto yo soy- responsable de él. Es una estructura que en nada se asemeja a la relación intencional que nos liga, en el conocimiento, al objeto -no importa de qué objeto se trate, aunque se un objeto humano-. La proximidad no remite a esta intencionalidad, en particular, no remite al hecho de que el otro me sea conocido. (Levinas, 1991, p. 98)

Estas condiciones se encuentran en conflicto, en el sentido en que la alteridad de la confianza en sociedad -es decir, la vida desde la mirada del otro sujeto- se ha relativizado y puesto en duda. Lo sostenido por Lévinas (1991) en la relación con el otro ser "se aproxima esencialmente a mí en tanto yo me siento ... responsable de él" (p. 98), pareciera ser, hoy, un factor de alta predeterminación cada vez que los sujetos deben responder con una imagen simulada o desvirtuada a las demandas de la vida en sociedad, a las convenciones, etc. Por este mismo hecho, ¿podremos generar y constituir un sistema de educación de una forma no punitiva?

\section{Lo complexus en el pensar pedagógico}

Es un hecho que muchos de los que piensan la educación chilena desde la filosofía, la teoría crítica y las ciencias de la educación concuerdan en que el escenario educativo chileno se encuentra en crisis, desde sus contenidos y prácticas hasta la formación de los sujetos. Pero qué significa preguntar por el pensar la verdad de la crisis educativa. En el entendimiento de esta problemática, surge un estado crisis y de replanteamiento, que pone a la hegemonía 
doi: http://dx.doi.org/10.15359/ree.21-2.21

URL: http://www.una.ac.cr/educare

CORREO: educare@una.cr

pensante, a las verdades filosóficas y a los principios fundamentales de las ciencias frente a frente con una realidad que se transforma rápidamente. Esta condición, precisamente, provoca incertidumbre, movimiento y transición hacia un nuevo marco referencial epistémico, es decir, al planteamiento de nuevas y esenciales preguntas para nuevas verdades. Por cierto, tenemos la sensación de que estamos frente a una crisis de los fundamentos del pensamiento (Morin, 1992) desde el momento en que se ha hecho necesaria una actitud dialógica entre la multi-disciplinar instalación del saber y el absolutismo de la tradición clásica para aprender su memoria; crisis que no es otra cosa que una vuelta a mirar el conocimiento desde el conocimiento, a la disciplina desde lo disciplinar y a la educación desde su propia originalidad. Con Morin (1999b):

Se trata de fortalecer la aptitud para interrogar y de vincular el saber a la duda, de desarrollar la aptitud para integrar el saber particular no solamente en un contexto global, sino también en su propia vida, la aptitud para plantearse los problemas fundamentales de su propia condición y de su propio tiempo. (Morin, 1999b, p. 126)

En este sentido, la tradición histórica del conocimiento científico, abordado desde el parcelamiento del saber y desbordado de la multidimensionalidad de los nuevos problemas (Morin, 1982), ha conducido a la incapacidad de pensar la crisis del conocimiento desde su instalación multifocal. Morín ha sostenido que mientras "más progresa la crisis, más progresa la incapacidad para pensar la crisis; cuanto más globales se vuelven los problemas, menos se piensa en ellos" (Morin, 1999b, p. 34). ¿Cómo entender el sentido que atribuye el autor a dicha incapacidad para pensar?, ¿qué origina dicha incapacidad? Al respecto, surgen nuevos cuestionamientos que pueden orientar la puesta en crisis de la verdad de la educación, y la veracidad del conocimiento y de las prácticas para la educación. Insisto con las interpelaciones: ¿Qué educar en tiempos globales?, ¿es protagónica la educación en la formación de una ciudadanía moderna? Por ende, en la sociedad del conocimiento la tecnologización de la educación y la ausencia de valoración social que el estudiantado da a los procesos pedagógicos, ¿plantea distancias y extrañezas con las prácticas formativas y educativas actuales?

Desde el ser pedagógico, la pregunta por las distancias y extrañezas de un sistema educativo en crisis no es sino la pregunta por la esencia del educar en el devenir de la historia contemporánea y del ser humano. En palabras de Humberto Giannini, "un rito irrenunciable, a través del cual se daban y se recibían aquellos saberes que hacen de la vida un valor y no un hecho" (Giannini, 1999-2000, p. 121). Por esto mismo, se debe comprender una organización educativa como un espacio para que se desarrolle lo que significa vivir en comunidad como sujeto social y no solo como una subjetividad. Una de las formas para buscar el desarrollo personal es encontrando el camino para ser líder en la formación de formadores, lo cual se inicia con la capacidad de dialogar en torno a sí, con nuestras propias acciones y experiencias de vida. Esto significa que debemos ser sujetos formadores de formadores y formadoras enseñando a enseñar a ser. 
La de-sublimación del educar en la tardo-modernidad ha obligado a preguntar: ¿qué se educa?, ¿quiénes se educan? y ¿para qué educar? Interrogantes que nos obligan a complementar la educación y la acción del pensar ya no desde como ¿qué pensar? sino más bien centrados en ¿cómo enseñar a pensar y a desarrollar la afección del sentir?

Preguntar desde el acto educativo no es sino también formular la pregunta por el origen de la acción educativa, por lo cual el acto educativo o el encuentro dialógico entre enseñantes y aprendices no cuestiona sino los fundamentos que hay que volver a mirar en la educación del siglo XXI. En este conjunto de interrogantes que interpelan a docentes y estudiantes, no tendrían otra motivación que transformar rigurosamente la educación como una problemática compleja que inquiete el conocimiento reflexivo y crítico referido al contexto y a los escenarios donde se desarrolla como proceso y permanencia de nuevas interrogantes, es decir, ¿quién transmite y educa?, ¿qué se forma y se transmite?, ¿a quién?, ¿con qué apoyo y condiciones?, ¿de qué forma?, ¿con qué propósito?, y finalmente, ¿con qué ideales sustentables para nuestra realidad cultural? Estas preguntas -en un devenir permanente- transportan en sí mismas el paradigma de la complejidad, ya que para Morin (2011) los tres soportes serían: un principio dialógico en tanto que todo fenómeno está en una relación de diálogo desde una multiunidualidad en el centro de una unidad, la recursividad organizacional entendida como proceso recursivo en el cual los productos y los efectos son, al mismo tiempo, causas y productores de aquello que los produce: auto-constitutivos, autoorganizadores y auto-productores) y, finalmente, el principio hologramático, donde la parte se ubica en el todo y el todo está en la parte -unitax múltiple (Morin, 1999b). Es en este sentido, el pensamiento complejo correspondería a una forma de reflexividad que vincula directamente sujetos y objetos de conocimiento para la comprensión de diversos contextos multidimensionales y relacionales. Esta noción es más que el contexto: es el conjunto de fenómenos que unen partes diversas de la experiencia y que permiten en su holístico tejido, entrelazar y entrecruzar una serie de condiciones biológicas, antropológicas, culturales, ambientales y creativas constituyentes del fenómeno de la vida. Esta manera de mirar el mundo y sus fenómenos se transforma en una experiencia de creación proyectiva, en la medida en que el sujeto despliega una relación con los objetos para su comprensión sensible y racional. La formación para el conocimiento de los diversos contextos relacionales radica también en el conocimiento sobre lo global. Lo global más que el contexto, es el conjunto que contiene partes diversas ligadas de manera inter-retroactiva y organizacional. De esta forma, una sociedad es más que un contexto, es un todo organizado y organizante del cual nos hacemos parte. El todo tiene cualidades o propiedades que no se encontrarían en aquellas, si estas se separaran las unas de las otras. Por esto, la comprensión de una mirada global instala la comprensión de las partes y en su extensión, la de los contextos. Para Morin (1999b): 
doi: http://dx.doi.org/10.15359/ree.21-2.21

URL: http://www.una.ac.cr/educare

CORREO: educare@una.cr

El ser humano como en los demás seres vivos, hay presencia del todo al interior de las partes: cada célula contiene la totalidad del patrimonio genético de un organismo policelular; la sociedad como un todo está presente en el interior de cada individuo en su lenguaje, su saber, sus obligaciones, sus normas. Así mismo, como cada punto singular de un holograma contiene la totalidad de la información de lo que representa, cada célula singular, cada individuo singular, contiene de manera holográmica el todo del cual hace parte y que al mismo tiempo hace parte de él. (p. 38)

Por último, una tercera gran interrogante relaciona la verdad del pensar (sentido e intencionalidad), con los propósitos fundamentales que el profesorado debiera considerar para una enseñanza que permita desarrollar la complejidad y el planteamiento de preguntas al interior de nuestros espacios educativos. Es posible interrogar que el origen de las distancias a la priorización de la educación radica en la pérdida de sentido de este mismo acto educativo, es decir, de la práctica docente. Será acaso poner en cuestión, por el origen de la acción y praxis, al interior de nuestros espacios educativos: una pregunta que, sin duda, interpela el quehacer docente. ¿Estamos en condiciones y disposiciones, el profesorado universitario, de preguntar por el sentido de la educación universitaria en tiempos de un extremo individualismo? Este problema plantea obviamente el trascendental rol de la universidad y de nuestras escuelas de pedagogía en el escenario chileno. En este sentido, la tarea como personal formador de profesionales del futuro es más compleja y más ardua. La puesta en marcha futura de profesorado competente, gestor de conocimientos e innovador en las formas de enseñar y también de aprender será el desafío del mañana.

Insisto con estas interpelaciones: ¿cómo enseñamos a nuestro alumnado a pensar?, ¿cómo enseñamos a preguntar? La convicción y la gran tarea está en formar estudiantes y futuro profesorado con un pensamiento crítico y reflexivo, con una capacidad para problematizar e ir en busca del conocimiento, es decir, de ser protagonistas y hacerse cargo de sus este y, por sobre todo, estimular y motivar a sus estudiantes para que sean gestores y gestoras del conocimiento. Todas estas condiciones transforman al personal docente en guía de ciertas habilidades, competencias y métodos que actualmente se encuentran muy olvidados en nuestras salas de clases. Desde esta perspectiva y visibilizando los riesgos de una educación tradicional, señala Morin (2001) que "como nuestra educación nos ha enseñado a separar, compartimentar, aislar y no a ligar los conocimientos, el conjunto de éstos constituye un rompecabezas ininteligible. Las interacciones, las retroacciones, los contextos, las complejidades que se encuentran entre las disciplinas se vuelven invisibles" (Morin, 2001, 42).

Me parece que un debate académico, dentro de nuestras universidades, podría constituir un conjunto coherente de respuestas a estas preguntas planteadas. Por de pronto, no se aspira sino a dialogar sobre nuestro quehacer como docentes; volver a mirar nuestras aspiraciones esenciales como seres humanos y, sobre todo, formar a profesionales que devuelvan al país lo que hemos despertado. 
Podemos pensar, también, en el aporte que nuestras escuelas de pedagogía pueden realizar en la construcción de nuevo paradigma de aprendizaje. Esta propuesta se consolida a partir de la convicción de que el espacio educativo es un lugar en el que tanto estudiantes como docentes comparten la experiencia de aprender de manera distinta y no distante. Por lo cual, considera reflexionar, pensar, creer e imaginar problemáticas del aprendizaje para hacer de la experiencia educativa una oportunidad real de conocer el cosmos individual y colectivo, aplicable a todas las experiencias o áreas de desarrollo.

En consideración a esta visión compleja de la enseñanza, se pueden sostener como principios metodológicos los siguientes:

- Los procesos formativos deben partir siempre de experiencias reales, contextuales y multidimensionales (lo bio-antropo-cultural del sujeto en su contexto local) para la valoración de la experiencia como un saber pedagógico. El carácter dialógico del encuentro entre lo experiencial del conocimiento y lo contenidista de las asignaturas permitirá integrar los procesos y saberes internalizados en la sucesiva de aprendizajes divergentes para el planteamiento de nuevos problemas.

- Toda enseñanza debe ser motivada por sus docentes desde una búsqueda inter-retroactiva y continua de los significados y sentidos de los aprendizajes involucrados para la instalación de nuevas miradas y nuevas preguntas. Esto genera la recursividad de los aprendizajes por parte de estudiantes y promueve nuevas formas de constituir los saberes disciplinares al interior de las salas de clases. Su potencia auto-constitutiva en la creación, auto-organizadora en la integración de saberes y auto-productora en la producción de conocimientos permitirá comprender que los contenidos y sus aprendizajes son, al mismo tiempo, estudiantes y saberes de aquella experiencia que les produce.

- La formación debe contemplar el aprendizaje como experiencia directa en el estudiantado, es decir, considerar este proceso como una actividad continua entre teoría y práctica, recordando el alcance hologramático en la relación del todo/partes y partes/todo de cualquier conocimiento disciplinar. En este contexto, se pueden establecer vínculos entre el conocimiento y los diversos saberes: relaciones entre las imágenes mentales (imaginario) y los conceptos que definen las cosas y los fenómenos educativos.

Esto nos hace pensar en estrategias metodológicas orientadas al estudiantado y profesorado para potenciar:

1. El hábito permanente de comunicar reflexivamente la realidad y todo lo que implica a nivel de comprensión, análisis e interpretación de los nuevos escenarios pedagógico-locales. 
doi: http://dx.doi.org/10.15359/ree.21-2.21

URL: http://www.una.ac.cr/educare

CORREO: educare@una.cr

2. La valoración del acto de preguntar y problematizar los contextos que se les presentan, es decir, incentivar el cuestionamiento, la duda y la incertidumbre como valores gestores de certezas y seguridades cognitivas, emocionales y valóricas.

3. La expresión y puesta en práctica de las ideas de cada quien. El pensar, problematizar, preguntar y reflexionar son formas de construir una propia visión del mundo, de la sociedad, de la organización y del ser humano, sujeta a debate y orientación por parte de un pensamiento complejo-creativo-productivo.

\section{La acción provoca-activa del pensamiento complejo}

La tarea de formar personal formador es más compleja y más ardua en nuestros tiempos. Esto nos hace pensar en una formación que altere las prácticas reproductivas de un modelo estratificado, según la condición social y ausente de participación social sustentable en el tiempo.

Por lo tanto, ¿qué pensar desde lo social en su complejidad? La universidad actual forma, en todo el mundo, una proporción demasiado grande de especialistas y; sin embargo, una gran parte de las actividades sociales, aquellas que la sociedad moderna requiere, necesita de personas capaces tanto de buscar nuevas estrategias para la apertura del conocimiento como de centrarse profundamente en los problemas a los que el progreso y la tecnologización de la vida nos enfrenta (Morin, 1999b). Si consideramos que la educación hacia lo social y desde lo social forma hombres y mujeres capaces de desenvolverse en diversos escenarios que abarcan desde las problemáticas socioculturales, económicas y políticas, hasta una formación para prevenir todo tipo de vulnerabilidades (intelectuales, emocionales, marginales, multiculturales, etc.) orientada para el desarrollo comunitario, ¿está la universidad moderna formando estudiantes en condiciones de devolverle al país estas precisas cualidades?

Me parece arriesgado sostener su veracidad cuando apreciamos lo contrario tanto a nivel público estatal como privado. Pienso que el problema sigue siendo la pregunta fundamental que nos hicimos en el comienzo de estos párrafos: ¿Pará qué educar?

Esta "poética educativa" orientada hacia la propiedad de lo íntimo de nuestra cultura necesita volver a pensar en su complejidad sobre: ¿qué es aprender?, ¿qué necesita el proceso de enseñanza aprendizaje en el contexto que nos toca vivir?, ¿qué significa ser docente hoy día en Chile? y, sobre todo, ¿qué ideal de ser humano queremos formar desde nuestra universidad? Mediante metodologías basadas en la experiencia, en el diálogo y en la multidimensionalidad de los contextos educativos, podemos acercarnos de un modo diferente al estudio del conocimiento, cambiando nuestro modo de estar y de situarnos en el sistema educacional, integrando a la actividad de aprender la dimensión lúdica y creativa en torno a la presentación de un mundo educativo para las verdaderas necesidades de país. 
En consecuencia, para cambiar el modo de educar, es necesario centrarse en la persona que se educa, conocer y estar en disposición de vincularnos, aplicar la empatía con el otro sujeto, legitimar y validar lo diverso y, en consecuencia, cambiar las maneras de pensar, sentir y actuar instaladas desde la cultura. Transformar dichas maneras de "ser" o "hacer" implica volver a mirar las verdades, compartir nuestras experiencias, construir y potenciar nuestros discursos. ¿Relacionamos nuestros saberes y conocimientos, nuestra ciencia y nuestra técnica con nuestros propios discursos? No es menor este tema, ya que hoy nos desarrollamos y convivimos con el amparo de un sistema marcado por la ideología neoliberal. Ideología por lo demás inserta en el paradigma moderno mecánico cartesiano, el cual ha permitido desarrollar importantes avances en las ciencias y la tecnología, pero también ha dejado enormes vacíos en la vida moderna y en nuestra propia existencialidad.

Perdimos nuestra humana relación con los demás individuos, con esos otros legítimos, con el entorno, con las creencias y reconocimiento de que también somos seres cosmológicos y partícipes del universo, perdimos la capacidad de escuchar, de preguntar, de escucharnos y de preguntarnos por nosotros mismos y nuestros propósitos.

Me permito nuevamente preguntar: ¿fueron esos antiguos y fundamentales ideales desde los discursos- los que cambiaron el devenir de la historia? Es decir, la conciencia de nuestra potencialidad humana, de nuestra identificación como seres humanos, exige cambios en los campos de producción educativa. Sin embargo, no han cambiado, aparentemente, en estos años, las reglas de juego ya que no sabemos qué juego estamos jugando, con quiénes los estamos jugando y para qué lo estamos jugando. ¿No será el momento en que realicemos una toma de conciencia efectiva?, ¿será la educación una vía legitimada para construír lo que esencialmente queremos habitar? La contradicción del sistema ha puesto en crisis todas las instituciones, todas las creencias y todas nuestras energías y esencias. Puso en crisis mi formación universitaria y pone también la de ustedes.

... debemos ver, ante todo eso que resulta del desarrollo técnico-económico. Muchas veces fue el individualismo, en el sentido de pérdida de las solidaridades tradicionales; el egocentrismo, que también destruye y olvida las solidaridades, y que se consagra únicamente a su propio interés; la pérdida de muchas aptitudes polivalentes del ser humano por la hiper-especialización de cada persona, la pérdida de muchas facultades por la adaptación y para enfrentar su destino. (Morin, 2008, p. 78)

¿Será necesaria la erudición y la valorización del conocimiento por el conocimiento el aprendizaje posmoderno en desmedro de la formación de un estudiantado crítico y reflexivo?

La toma de conciencia parte desde el momento en que reconocemos nuestra propia esencialidad, desde el momento en que aceptamos nuestra historia personal, desde cómo 
doi: http://dx.doi.org/10.15359/ree.21-2.21

URL: http://www.una.ac.cr/educare

CORREO: educare@una.cr

nos miramos internamente y miramos a nuestro alrededor, desde el reconocimiento de seres formados en la libertad, es decir, capaces de tomar decisiones que nos comprometan en la voluntad creciente de nuestras necesidades más internas, condiciones que nos permitirán visualizar qué juego estamos jugando, reconocer con quiénes estamos jugando, y por sobre todo, invitar a quienes estén dispuestos a cambiar las reglas del juego.

La educación se construye y se habita para nuevas prácticas. Por tanto, ¿cómo construimos y habitamos la educación? Pregunta que se funda en el conocimiento, en la razón, en la sensibilidad y en la instalación por nuestra parte como profesorado de nuevos valores, tales como el valor de la incertidumbre, de la duda, de la capacidad de criticar fundadamente, de la capacidad de cuestionar, de preguntar, de conflictuar nuestra propia realidad; valores, por lo demás, que complementarían y concretarían en su aplicabilidad la tradicional enseñanza academicista.

El juicio crítico, el compromiso, la experiencia personal, la libertad creadora y la capacidad para teorizar, entre otras cualidades, es lo que debemos modelar el personal académico para contribuir al establecimiento de una nueva mentalidad, que implique definitivamente superar los obstáculos que ocultan al ser humano y lo transforman en una mercancía. La formación para la toma de conciencia no margina el contenido ni menos el conocimiento, son sus mejores herramientas para asumir nuestro pasado, criticar el presente y definirnos hacia el futuro. Morin (1983) señala que ver lo que todo el mundo ha visto necesita la nueva concepción de pensar lo que nadie ha pensado y quizás percibido. De este modo, todo descubrimiento, empezando por el de una cosa visible para todos y todas, es una conquista cognitiva que comporta invención y creación.

Me parece que la totalidad de intelectuales que pensamos la educación en Chile, llegamos a similares conclusiones: Nuestra patria debe sustentar sus transformaciones técnicas, sociales y políticas desde la formación educativa inicial. Ese es el desafío de pensar la construcción de una escuela y universidad desde la complejidad. Tomemos conciencia del rol protagónico que tanto estudiantes como docentes debemos asumir para transformar nuestra historia educativa. La invitación está hecha y la sala de clases es su lugar.

\section{Conclusiones}

La enseñanza tradicional ha promovido la separación de las disciplinas y de los objetos con su entorno. En esta separación, no se logra relacionar lo que realmente se encuentra unido y entretejido en un contexto donde la inteligencia no sabe hacer otra cosa que separar. De esta forma, las maneras de conocer y, en consecuencia, el conocimiento pertinente para los contextos multivariables deben dejar en evidencia la relación que posee el conocimiento con su propio sentido de existencia, en tanto que la enseñanza debería reconocer, en el conocimiento, su propia humanidad al situarlo en el mundo y asumirlo como posibilidad de transformación y saber. 
Chile necesita incorporar, en su sistema educativo, la permanente reflexividad (pensar complejamente los entornos pedagógicos) para promover el pensamiento creativo, crítico y consciente a través del desarrollo de procesos cognitivos como la percepción, la interpretación, la comprensión, el análisis, el desarrollo de la intuición, la sensibilidad creadora y la corporización de las experiencias como sustento del sentido de los aprendizajes en el estudiantado. Por este hecho, el principio dialógico de la complejidad se torna fundamental para complementar procesos de enseñanza y aprendizajes efectivos al interior de las salas de clases. En el descubrimiento de lo sensible y del sentir el fenómeno educativo, se logrará desarrollar paulatinamente cuestiones como la motivación por aprender desde el riesgo para lo incierto; la construcción de nuevos conocimientos; el planteamiento de nuevos problemas para nuevas preguntas; la aceptación de la legitimidad del otro ser; la valoración de la diversidad; el descubrimiento de un pensamiento crítico en el trabajo autónomo y el desarrollo del pensamiento holístico, entre tantas otras posibilidades reales al interior de las aulas chilenas.

Tanto los conocimientos contextuales como locales nos remiten a un conocimiento más trascendental y particular: el conocimiento en la complejidad del sí-mismo/misma en la formación escolar y universitaria. Esta dialógica permanente deberá tener, como base, la valoración de la experiencia como un saber recíproco entre lo uno y la multidimensionalidad de los fenómenos. La educación puede generar una verdadera cultura por aprender y enseñar el sentido real de la experiencia, de compartir el conocimiento y de construir en conjunto nuevas miradas para nuevos diálogos.

En la perspectiva del pensamiento complejo, debemos avanzar hacia la re-articulación de las disciplinas en su transdisciplinariedad, pero lo más importante es mantener el espíritu crítico que permite descubrir sentidos nuevos de las cosas y sus relaciones. La inter y trans-disciplinaridad nos permite ver desde nuevas maneras la realidad, ya que nos abre la perspectiva para nuevos lenguajes que no conocíamos y que son tan válidos como los aceptados socialmente. En la formación pedagógica, las relaciones con el mundo local y las nuevas prácticas metodológicas permiten explorar mundos y contextos plenos de significados culturales, que amplían las indagaciones para investigar y crear nuevas formas de representación que transitan entre la cultura y la vida.

Transdisciplina y complejidad en la educación están estrechamente unidas como formas de pensamiento relacional, y como interpretaciones del conocimiento desde la perspectiva de la existencia humana.

En consecuencia, esta propuesta para el desarrollo del pensamiento complejo en las aulas de formación pedagógica, debiera contemplar los siguientes aspectos:

1. Clases destinadas a la revisión de teorías, desarrollo de matrices conceptuales y precisión discursiva-argumentativa, las cuales tendrán significado siempre y cuando se complementen con discusiones reflexivas y relaciones multidimensionales de los saberes. 
doi: http://dx.doi.org/10.15359/ree.21-2.21

URL: http://www.una.ac.cr/educare

CORREO: educare@una.cr

2. Clases destinadas al descubrimiento de experiencias desde la experiencia de los sujetos involucrados (docentes, estudiantes, comunidad, etc.). Una valoración permanente de la observación del medio y de los medios como manera de introducirse en el saber, una aceptación del error responsable como posibilidad de aprendizaje divergente y comprensión de lo incomprendible.

3. Clases orientadas a aprender a través del hacer con otros seres y en el propio descubrimiento de las potencialidades creativas en la puesta en práctica de los saberes disciplinares para la formación de personal formador.

4. Identificación de lo local/global/local para el aprendizaje cultural, artístico y social. Desde el pensamiento creativo, estético, reflexivo y crítico se podrá instalar una materialidad contextual que desarrollará nuevos significados para la ampliación de nuestra conciencia pedagógica.

5. Hacer pensar la complejidad como una acción provoca-activa, que produzca sentido de pertenencia con lo educativo local. La tarea como docentes e intelectuales es más compleja en nuestros tiempos. La puesta en marcha futura de profesionales competentes, que generen conocimientos e innovación en las formas de producir será el desafío del mañana.

Una educación centrada en la complejidad tiene la misión de incentivar la aptitud para problematizar, la aptitud para interrogar e interrogarse, la aptitud para contextualizar y, en consecuencia, la toma de conciencia y voluntad de enfrentar el gran desafío de lo complexus en la modernidad y en nuestras salas de clases. Quizás lo más complejo de la complejidad sea el no dar el paso para un cambio más complejo.

La pregunta: ¿qué significa pensar la complejidad desde el hacer pedagógico?, sitúa esta forma de mirar el mundo en la diversidad de posibilidades teóricas y prácticas para visualizar el pensar formativo en consideración a los actuales modelos, acciones y tipos de enseñanza, permitiendo un cuestionamiento sobre nuestra propia realidad, la transformación en el pensamiento social, la reflexión crítica del cuerpo académico y del sentido educativo.

\section{Referencias}

Derrida, J. y Ferraris, M. (2009). El gusto del secreto. Buenos Aires: Amorrortu.

Giannini, H. (1999-2000). Crítica y reflexión. Revista enfoques educacionales, 2(2), 121134. Recuperado de http://www.facso.uchile.cl/publicaciones/enfoques/04/docs/ enfoques 04 1999.pdf 
Lévinas, E. (1991). Ética e infinito. Madrid: La balsa de la Medusa.

Maturana, H. (2007). Emociones y lenguaje en educación y política. Santiago: Comunicaciones Noreste.

Moraes, M. C. y De La Torre, S. (2002). Sentipensar bajo la mirada autopoiética o cómo reencantar creativamente la educación. Creatividad y sociedad, 2, 41-56. Recuperado de http://www. waldorfcolombia.org/seccns/Reencantando.pdf

Morin, E. (1982). Ciencia con conciencia. Barcelona: Anthropos.

Morin, E. (1983). El método III. El conocimiento del conocimiento. Madrid: Cátedra.

Morin, E. (1992). El método 4: Las ideas. Madrid: Cátedra.

Morin, E. (1998). Complejo de amor. Gazeta de antropología, 14(1), 1-6. Recuperado de http:// www.ugr.es/ pwlac/G14 01Edgar Morin.pdf

Morin, E. (1999a). El desafío del siglo XXI. Unir los conocimientos. La Paz: Plural Editores.

Morin, E. (1999b). La cabeza bien puesta. Buenos Aires, Argentina: Nueva Visión.

Morin, E. (2001). Los siete saberes necesarios para la educación del futuro. Buenos Aires, Argentina: Nueva Visión.

Morin, E. (2008). Estamos en un Titanic. Recuperado de http://www.edgarmorin.org/images/ publicaciones/edgar-morin-estamos-en-un-titanic.pdf

Morin, E. (2011). Introducción al pensamiento complejo. Barcelona: Gedisa.

Nancy, J.-L. (1993). The birth to presence. Stanford: Stanford University Press.

Pineau, G (2009). Estrategia universitaria para la transdisciplinariedad y la complejidad. Revista Visión Docente Con-Ciencia, 8(48), 5-17. Recuperado de http://www.ceuarkos.com/Vision docente/revistas/48/ESTRATEGIA\%20UNIVERSITARIA.pdf 